\title{
THE CONVEX INTERSECTION BODY OF A CONVEX BODY
}

\author{
MATHIEU MEYER \\ Université Paris-Est - Marne-la-Vallée, Laboratoire d'Analyse et de Mathématiques Appliquées \\ (UMR 8050), Cité Descartes, 5 Bd Descartes, Champs-sur-Marne, 77454 Marne-la-Vallée cedex 2, France \\ e-mail: mathieu.meyer@univ-mlv.fr \\ and SHLOMO REISNER $\dagger$ \\ Department of Mathematics, University of Haifa, Haifa 31905, Israel \\ e-mail: reisner@math.haifa.ac.il
} (Received 2 April 2010; revised 29 September 2010; accepted 30 November 2010;
first published online 10 March 2011)

\begin{abstract}
Let $L$ be a convex body in $\mathbb{R}^{n}$ and $z$ an interior point of $L$. We associate with $L$ and $z$ a new, convex and centrally symmetric, body $C I(L, z)$. This generalizes the classical intersection body $I(L, z)$ (whose radial function at $u \in S^{n-1}$ is the volume of the hyperplane section of $L$ through $z$, orthogonal to $u)$. $C I(L, z)$ coincides with $I(L, z)$ if and only if $L$ is centrally symmetric about $z$. We study the properties of $C I(L, z)$.
\end{abstract}

2010 Mathematics Subject Classification. 52A20.

1. Introduction. Let $L$ be a convex body in $\mathbb{R}^{n}$ containing 0 in its interior. The intersection body $I(L)$ of $L$, defined by its radial function $\rho_{I(L)}$ on the sphere $S^{n-1}$, which is

$$
\rho_{I(L)}(u)=\operatorname{vol}\left(L \cap u^{\perp}\right)
$$

and the cross-section body $C(L)$ of $L$, defined by

$$
\rho_{C(L)}(u)=\max _{t} \operatorname{vol}\left(\left(L \cap\left(t u+u^{\perp}\right)\right)\right.
$$

are not, in general, convex bodies, although they are identical and, moreover, convex in the case when $L$ is centrally symmetric. This follows from Brunn-Minkowski theorem (see [21, p. 309]) and from Busemann's theorem (see [3]). We introduce here a new convex body associated with $L$, generalizing both the intersection body and the crosssection body. More precisely, we define the convex intersection body $C I(L)$ of $L$ by its radial function

$$
\rho_{C I(L)}(u)=\min _{z \in P_{u}\left(L^{* g(L)}\right)} \operatorname{vol}\left(\left[P_{u}\left(L^{* g(L)}\right)\right]^{* z}\right)
$$

†Both authors were supported in part by the France-Israel Research Network Program in Mathematics contract \#3-4301. 
where $g(L)$ denotes here the centroid of $L$, and if $E$ is a linear subspace of $\mathbb{R}^{n}$ and $M$ is a convex body in $E$, we define for $z \in E$,

$$
M^{* z}=\{y \in E ;\langle y-z, x-z\rangle \leq 1 \text { for every } x \in M\} .
$$

Thus (1) means: first apply duality with respect to the point $g(L)$, then project onto $u^{\perp}$, finally apply duality with respect to $z$ and minimize the $(n-1)$-dimensional volume over $z$.

In Section 2, we shall attach to any convex body $K$ in $\mathbb{R}^{n}$ a body $J(K)$ constructed with the help of its projections, and prove that it is always convex. In Section 3 , we prove that $C I(L)$ is convex, and we study the inclusions

$$
C I(L) \subset I(L) \subset C(L)
$$

when $g_{L}=0$. Finally, in Section 4, a few open problems are listed, with proposed ideas concerning some of them.

NotATions. For $x, y \in \mathbb{R}^{n}$, we denote by $\langle x, y\rangle$ the canonical scalar product in $\mathbb{R}^{n}$, $|x|$ denotes the Euclidean norm defined by it. If $u$ and $v$ are two non-zero vectors in $\mathbb{R}^{n}$ that are not orthogonal to one another, we define $\Pi_{w, u^{\perp}}: \mathbb{R}^{n} \rightarrow u^{\perp}$ to be the projection parallel to $w$ onto $u^{\perp}=\left\{x \in \mathbb{R}^{n} ;\langle x, u\rangle=0\right\}$, we denote

$$
P_{u}=\Pi_{u, u^{\perp}}
$$

If $L$ is a subset of $\mathbb{R}^{n}$, let $[L]$ be the affine subspace of $\mathbb{R}^{n}$ that it spans. If $B$ is a convex subset of $\mathbb{R}^{n}$, we denote its $k$-dimensional volume by $\operatorname{vol}(B)$ (where $k=\operatorname{dim}[B]$ ). By $\overline{\operatorname{conv}}(A)$, we denote the closed convex hull of $A$. to $z$ by

For $L$ a convex set in $\mathbb{R}^{n}$ and $z \in[L]$, we denote the polar body of $L$ with respect

$$
L^{* z}=\{y \in[L] ;\langle y-z, x-z\rangle \leq 1 \text { for every } x \in L\} .
$$

It is well known that the function $z \rightarrow \operatorname{vol}\left(L^{* z}\right)$ is strictly convex on the relative interior of $L$ and tends to $+\infty$ as $z$ approaches the realtive boundary of $L$ in $[L]$ (see $[17,20])$. It follows that it reaches its minimum at a unique point $s(L) \in \operatorname{int}(L)$. This point is called the Santaló point of $L$. We shall denote

$$
L^{* s}:=L^{* s(L)} .
$$

Moreover, $s(L)$ is also characterized as the unique point $z \in \operatorname{int}(L)$, which is the centroid of $L^{* z}$ (see [20] and also [21, p. 419]). Let us denote by $g(M)$ the centroid of a convex body $M$ in $[M]$, and set

$$
M^{* g}=M^{* g(M)}
$$

One has

$$
L^{* s}=M \text { if and only if } M^{* g}=L .
$$

Observe that, in general, $s(L) \neq g(L)$ (see the recent [18], where a lower bound to how far apart these two points can be is given). Finally if $0 \in \operatorname{int}(M)$, we shall write $M^{*}=M^{* 0}$, so that $M^{* x}=x+(M-x)^{*}$ for every $x \in \operatorname{int}(M)$. 
We adopt the following notation: if $K$ is a star body with respect to 0 , we denote

$$
\|x\|_{K}=\inf \{\lambda>0 ; x \in \lambda K\}
$$

to be the gauge of $K$. Then for $u \in S^{n-1}$,

$$
\rho_{K}(u)=\frac{1}{\|u\|_{K}}
$$

is the radial function of $K$.

\section{A convexity theorem.}

THEOREM 1. If $K$ is a convex body in $\mathbb{R}^{n}$, let $N_{K}: \mathbb{R}^{n} \rightarrow \mathbb{R}_{+}$be defined by the formula

$$
N_{K}(u)=\frac{1}{\operatorname{vol}\left(\left(P_{u} K\right)^{* s}\right)}=\frac{1}{\min _{z \in u^{\perp}} \operatorname{vol}\left(\left(P_{u} K\right)^{* z}\right)} \text { for } u \in S^{n-1},
$$

and extended to all $\mathbb{R}^{n}$ by $N_{K}(r u)=r N_{K}(u)$ for $r \geq 0$ and $u \in S^{n-1}$. Then $N_{K}$ is a norm on $\mathbb{R}^{n}$.

Before proving Theorem 1, we need some preliminary results.

Definition . Let $v \in S^{n-1}, B$ be a bounded subset of $\mathbb{R}^{n}$ and $V: B \rightarrow \mathbb{R}$ be a bounded map. The shadow system $\left(L_{t}\right), t \in[a, b]$ of convex bodies in $\mathbb{R}^{n}$, with direction the vector $v$, with basis the set $B$ and with speed the function $V$, is the family of convex bodies

$$
L_{t}=\overline{\operatorname{conv}}\{b+t V(b) v ; b \in B\}, \text { for } t \in[a, b] .
$$

For the sake of completeness, we prove the following result, which appears in [23] and is used, for example, in $[\mathbf{4}, \mathbf{5}]$.

Proposition 2. Let $K$ be a convex body in $\mathbb{R}^{n}$. Then, for $u, v \in S^{n-1}$, such that $\langle u, v\rangle=0$, the family $L_{t}=\Pi_{u+t v, u^{\perp}} K, t \in \mathbb{R}$, is a shadow system of convex bodies in $u^{\perp}$, in the direction $v$.

Proof. To simplify notation, one may suppose that $u=e_{n}$ and $v=e_{n-1}$, where $e_{1}, \ldots, e_{n}$ is an orthonormal basis of $\mathbb{R}^{n}$ (we shall write $\mathbb{R}^{j}$ for $\left[e_{1}, \ldots, e_{j}\right]$ ). Then, for all $t \in \mathbb{R}$,

$$
\begin{aligned}
\Pi_{u+t v, u^{\perp}} K & =\left\{X+z e_{n-1} ; X \in \mathbb{R}^{n-2}, X+z e_{n-1}+r\left(e_{n}+t e_{n-1}\right) \in K \text { for some } r \in \mathbb{R}\right\} \\
& =\left\{X+z e_{n-1} ; X \in \mathbb{R}^{n-2}, X+(z+r t) e_{n-1}+r e_{n} \in K \text { for some } r \in \mathbb{R}\right\} \\
& =\left\{X+(x-r t) e_{n-1} ; X \in \mathbb{R}^{n-2}, r \in \mathbb{R} \text { such that } X+x e_{n-1}+r e_{n} \in K\right\} \\
& =\left\{U-r t e_{n-1} ;(U, r) \in P_{u} K \times \mathbb{R} \text { such that } U+r e_{n} \in K\right\} .
\end{aligned}
$$

For $U \in P_{u} K$, define

$$
\mathcal{I}(U)=\left\{r \in \mathbb{R} ; U+r e_{n} \in K\right\} .
$$

Then $\mathcal{I}(U)=[a(U), b(U)]$ is a closed interval of $\mathbb{R}$. Define also $x(U) \in \mathbb{R}$ such that

$$
\left\langle U-x(U) e_{n-1}, e_{n-1}\right\rangle=0,
$$


and let

$$
D_{1}=\left\{U \in P_{u} K ; x(U) \in \mathbb{Q}\right\} \text { and } D_{2}=\left\{U \in P_{u} K ; x(U) \in \mathbb{R} \backslash \mathbb{Q}\right\} .
$$

Define $V: P_{u} K \rightarrow \mathbb{R}$ by

$$
v(U)=-b(U) \text { if } U \in D_{1} \text { and } v(U)=-a(U) \text { if } U \in D_{2} .
$$

By the continuity of the two concave functions $-a, b: P_{u}(K) \rightarrow \mathbb{R}$, it is easy to see that for every $t \in \mathbb{R}$

$$
\Pi_{u+t v, u^{\perp}} K=\overline{\operatorname{conv}}\left\{U+t V(U) e_{n} ; U \in P_{u} K\right\} .
$$

REMARK. The converse assertion of Proposition 2 is true : every shadow system $L_{t}$ in $\mathbb{R}^{n}$ can be represented as $L_{t}=\Pi_{u+t v, u^{\perp}}(K)$ with an appropriate convex body $K \subset \mathbb{R}^{n+1}$ and $u, v \in S^{n}$. This was shown, for example, in [4].

The following result was proved in [16].

THEOREM 3. Let $t \in[a, b] \rightarrow L_{t}$ be a shadow system in $\mathbb{R}^{n}$, then the function $\phi:$ $\mathbb{R}^{n} \rightarrow \mathbb{R}$, defined by the formula

$$
\phi(t)=\frac{1}{\operatorname{vol}\left(\left(L_{t}\right)^{* s}\right)}=\frac{1}{\min _{z} \operatorname{vol}\left(\left(L_{t}\right)^{* z}\right)},
$$

is convex.

Lemma 4. Let $N: \mathbb{R}^{n} \rightarrow \mathbb{R}_{+}$satisfy

- $N(x)>0$ for $x \neq 0$,

- $N(\alpha x)=|\alpha| N(x)$ for every $\alpha \in \mathbb{R}$ and $x \in \mathbb{R}^{n}$,

- for all $u, v \in S^{n-1}$ such that $\langle u, v\rangle=0, t \mapsto N(u+t v)$ is convex on $\mathbb{R}$.

Then $N$ is a norm on $\mathbb{R}^{n}$.

Proof. Let us show that $N(x+y) \leq N(x)+N(y)$ for every $x, y \in \mathbb{R}^{n} \backslash\{0\}$. Let $\alpha=\left|\frac{x}{|x|}+\frac{y}{|y|}\right|$ and $\beta=\left|\frac{x}{|x|}-\frac{y}{|y|}\right|, u=\frac{1}{\alpha}\left(\frac{x}{|x|}+\frac{y}{|y|}\right)$ and $v=\frac{1}{\beta}\left(\frac{x}{|x|}-\frac{y}{|y|}\right)$. We may suppose that $\alpha \neq 0$ and $\beta \neq 0$. Then $u, v \in S^{n-1},\langle u, v\rangle=0$ and

$$
x=\frac{|x|}{2}(\alpha u+\beta v), \quad y=\frac{|y|}{2}(\alpha u-\beta v) .
$$

We get

$$
\begin{aligned}
N(x+y) & =\frac{\alpha(|x|+|y|)}{2} N\left(u+\frac{\beta(|x|-|y|)}{\alpha(|x|+|y|)} v\right) \\
& =\frac{\alpha(|x|+|y|)}{2} N(u+(\lambda t+(1-\lambda) s) v),
\end{aligned}
$$


where $\lambda=\frac{|x|}{|x|+|y|}, t=\frac{\beta}{\alpha}$ and $s=-\frac{\beta}{\alpha}$. Under the assumption of the lemma, we get

$$
\begin{aligned}
N(x+y) & \leq \frac{\alpha(|x|+|y|)}{2}(\lambda N(u+t v)+(1-\lambda) N(u+s v)) \\
& =\frac{\alpha}{2}\left(|x| N\left(u+\frac{\beta}{\alpha} v\right)+|y| N\left(u-\frac{\beta}{\alpha} v\right)\right)=N(x)+N(y) .
\end{aligned}
$$

Proof of Theorem 1. In view of Lemma 4, we need to prove that $t \rightarrow g_{u, v}(t)=$ $N(u+t v)$ is convex, whenever $u, v \in S_{n-1}$ satisfy $\langle u, v\rangle=0$. It is easy to see that for any $t \in \mathbb{R}, P_{u+t v} K$ is an affine image of $\Pi_{u+t v, u^{\perp}} K$ and satisfies

$$
\operatorname{vol}\left(P_{u+t v} K\right)=\frac{1}{\sqrt{1+t^{2}}} \operatorname{vol}\left(\Pi_{u+t v, u^{\perp}} K\right) .
$$

Hence

$$
\left.\min _{z \in\{u+t v\}^{\perp}} \operatorname{vol}\left(\left(P_{u+t v} K\right)^{* z}\right)=\sqrt{1+t^{2}} \min _{z \in u^{\perp}} \operatorname{vol}\left(\left(\Pi_{u+t v, u^{\perp}} K\right)^{* z}\right)\right)
$$

It follows that

$$
N(u+t v)=\frac{|u+t v|}{\left.\min _{z \in\{u+t v\}^{\perp}} \operatorname{vol}\left(\left(P_{u+t v} K\right)^{* z}\right)\right)}=\frac{1}{\min _{z \in u^{\perp}} \operatorname{vol}\left(\left(\Pi_{u+t v, u^{\perp}} K\right)^{* z}\right)} .
$$

Now by Proposition 2, $t \rightarrow \Pi_{u+t v, u^{\perp}} K$ is a shadow system on $\mathbb{R}$ and thus by Theorem 3, $g_{u, v}$ is convex on $\mathbb{R}$.

REMARKS. (1) If $K$ is centrally symmetric (and centred at 0 ), then all its projections $P_{u} K$ are centrally symmetric (and centred at 0 ) so that

$$
\left.\left.\min _{z \in u^{\perp}} \operatorname{vol}\left(P_{u} K\right)^{* z}\right)=\operatorname{vol}\left(P_{u} K\right)^{* 0}\right)=\operatorname{vol}\left(K^{* 0} \cap u^{\perp}\right) .
$$

Under this hypothesis, we proved that $u \rightarrow \frac{1}{\operatorname{vol}\left(K^{* 0} \cap u^{\perp}\right)}$ is the restriction to $S^{n-1}$ of a norm on $\mathbb{R}^{n}$. This is Busemann [3] theorem on the sections of convex centrally symmetric bodies, applied to $K^{*}$.

(2) For every convex body $K$ in $\mathbb{R}^{n}$, Theorem 1 defines a centrally symmetric convex body $J(K)$ in $\mathbb{R}^{n}$ by

$$
J(K)=\left\{x \in \mathbb{R}^{n} ; N_{K}(x) \leq 1\right\} .
$$

Notice that for every $x_{0} \in \mathbb{R}^{n}, J\left(K+x_{0}\right)=J(K)$ and that for $A: \mathbb{R}^{n} \rightarrow \mathbb{R}^{n}$ a linear isomorphism, we have

$$
J((A K))=|\operatorname{det}(A)|\left(A^{*}\right)^{-1}(J(K)) .
$$

(3) If $n=2$ and if $R$ is the rotation by angle $\pi / 2$ in $\mathbb{R}^{2}$, then

$$
\operatorname{vol}\left(P_{u} K\right)=h_{K}(R u)+h_{K}(-R u)=h_{K}(R u)+h_{-K}(R u),
$$


so that

$$
J(K)=\frac{1}{4} R(K-K)
$$

3. The convex intersection bodies $I C(L, z)$ of a convex body $L$. Let $L$ be a convex body in $\mathbb{R}^{n}$. For a point $z \in \operatorname{int}(L)$, the intersection body $I(L, z)$ of $L$ with respect to $z$ is the centrally symmetric star body in $\mathbb{R}^{n}$ whose radial function $\rho_{I(L, z)}$ is given for $u \in S^{n-1}$ by

$$
\rho_{I(L, z)}(u)=\operatorname{vol}(\{x \in L ;\langle x-z, u\rangle=0\})=\operatorname{vol}\left(L \cap\left(z+u^{\perp}\right)\right) .
$$

The body $C(L)$ is the star body in $\mathbb{R}^{n}$ defined by its radial function

$$
\rho_{C(L)}(u)=\max _{x \in L} \operatorname{vol}\left(L \cap\left(x+u^{\perp}\right)\right) .
$$

Of course, one has $I(L, z) \subset C(L)$ for every $z \in \operatorname{int}(L)$. It was proved in [13] that these bodies coincide if and only if $L$ is centrally symmetric about $z$ (the 'if' part follows easily from Brunn-Minkowski theorem). We define now the convex intersection body of $L$ with respect to $z \in \operatorname{int}(L)$, which we denote by $C I(L, z)$, by

$$
C I(L, z)=J\left(L^{* z}\right) .
$$

When $z=g(L)$ is the centroid of $L$, we shall denote $C I(L)=C I(L, g(L))$. The radial function of $C I(L, z)$ is thus given for $u \in S^{n-1}$ by

$$
\rho_{C I(L, z)}(u)=\min _{x \in u^{\perp}} \operatorname{vol}\left(\left(P_{u}\left(L^{* z}\right)\right)^{* x}\right)=\operatorname{vol}\left(\left(P_{u}\left(L^{* z}\right)\right)^{* S}\right) .
$$

In view of Theorem 1 , one has

THEOREM 5. Let L be a convex body. Then for every $z \in \operatorname{int}(L), C I(L, z)$ is a centrally symmetric convex body such that $C I(L, z) \subset I(L, z)$.

REMARKS. (1) It is easy to see that one has for every one-to-one affine map $A: \mathbb{R}^{n} \rightarrow \mathbb{R}^{n}, I(A L, A z)=|\operatorname{det}(A)| A^{*-1}(I(L, z))$, as well as

$$
C I(A L, A z)=|\operatorname{det}(A)| A^{*-1}(C I(L)) .
$$

(2) In the case $n=2$, for $u \in S^{1}$, denoting by $R$ the rotation around 0 of angle $\pi / 2$, one has

$$
\begin{aligned}
\|u\|_{C(L)} & =\|R u\|_{K-K} \\
& \leq\|u\|_{I(L, z)}=\left(\frac{1}{\|R u\|_{K-z}}+\frac{1}{\left.\|-R u\|_{K-z}\right)}\right)^{-1} \\
& \leq\|u\|_{C I(L, z)}=4\left(\|R u\|_{K-z}+\|-R u\|_{K-z}\right) .
\end{aligned}
$$

(3) The inclusion $C I(L, z) \subset I(L, z)$ is exact in the sense that their boundaries touch; there always exists $u \in S^{n-1}$ such that

$$
\operatorname{vol}\left(L \cap\left(z+u^{\perp}\right)\right)=\operatorname{vol}\left(\left(P_{u}\left(L^{* z}\right)\right)^{* s}\right) .
$$


As a matter of fact, this equality means that the centroid of $L \cap\left(z+u^{\perp}\right)$ in $z+u^{\perp}$ is at $z$. To see that such $u$ exists, define $\phi: S^{n-1} \rightarrow \mathbb{R}$ by

$$
\phi(v)=\operatorname{vol}(\{x \in L ;\langle x-z ; v\rangle \geq 0\}) .
$$

Since $\phi$ is continuous, it reaches its maximum at some point $u \in S^{n-1}$. Then, by [15], $z$ is the centroid of $L \cap\left(z+u^{\perp}\right)$. See also [10].

(4) It was proved by Grünbaum ([10, Section 6.2]) that for every convex body $L \in \mathbb{R}^{n}$, there exists some $z_{0} \in \operatorname{int}(L)$ such that $(n+1)$ different hyperplanes through $z_{0}$, with normals $u_{1}, \ldots, u_{n+1}$, satisfy that $z_{0}$ is the centroid of $L \cap\left(z+u_{i}^{\perp}\right)$. For this $z_{0}$, the boundaries of $C I\left(L, z_{0}\right)$ and of $I\left(L, z_{0}\right)$ have at least $2(n+1)$ contact points.

(5) We have seen above that, in the case when $L$ is centrally symmetric about $z$, $C I(L, z)=I(L, z)$ and Theorem 5 is nothing else but the classical Busemann's theorem [1]. Conversely, the following result holds.

Proposition 6. One has $C I(L, z)=I(L, z)$ if and only if $L$ is centrally symmetric about $z$.

It is a consequence of the following lemma.

Lemma 7. Let L be a convex body and $z \in L$. Suppose that $z$ is the centroid of every hyperplane section of $L$ through itself. Then $L$ is centrally symmetric about $z$.

Proof. Fix some $z_{0} \in \operatorname{int}(L), z_{0} \neq z$, and define $F: \mathbb{R}^{n} \rightarrow \mathbb{R}$ by

$$
F(y)=\operatorname{vol}\left(\left\{x \in L-z_{0} ;\langle x, y\rangle \geq 1\right\}\right) .
$$

By [15], $F$ is $C^{1}$ on $\{F>0\}=\mathbb{R}^{n} \backslash\{0\}$ and one has for $y \neq 0$

$$
\begin{aligned}
\nabla F(y) & =\langle\nabla F(y), y\rangle g\left(\left\{w \in L-z_{0} ;\langle w, y\rangle=1\right\}\right) \\
& =\langle\nabla F(y), y\rangle\left(g\left(\left\{x \in L ;\left\langle x-z_{0}, y\right\rangle=1\right\}\right)-z_{0}\right) .
\end{aligned}
$$

Let $H$ be the affine hyperplane in $\mathbb{R}^{n}$ defined by

$$
H=\left\{y \in \mathbb{R}^{n} ;\left\langle z-z_{0}, y\right\rangle=1\right\} .
$$

If $y \in H$, the hyperplane $\left\{x \in \mathbb{R}^{n} ;\left\langle x-z_{0}, y\right\rangle=1\right\}$ passes through $z$, so that by the hypothesis, one has

$$
g\left(\left\{w \in L ;\left\langle x-z_{0}, y\right\rangle=1\right\}\right)=z,
$$

thus, by (2) we get

$$
\nabla F(y)=\langle\nabla F(y), y\rangle\left(z-z_{0}\right)
$$

Now if $y, y^{\prime} \in H$, one has

$$
\left\langle y^{\prime}-y, z-z_{0}\right\rangle=0 \text { and for every } t \in \mathbb{R},(1-t) y^{\prime}+t y \in H
$$

so that

$$
\left.F\left(y^{\prime}\right)-F(y)=\int_{0}^{1}\left\langle y^{\prime}-y, \nabla F\left((1-t) y+t y^{\prime}\right)\right)\right\rangle d t=0
$$


Thus, $F$ is equal to some constant $c$ on $H$. Define a function $G: S^{n-1} \rightarrow \mathbb{R}$ by

$$
G(u)=\operatorname{vol}\{x \in L ;\langle x-z, u\rangle \geq 0\} .
$$

and let

$$
U=\left\{u \in S^{n-1} ;\left\langle u, z-z_{0}\right\rangle>0\right\} .
$$

Then $u \rightarrow y(u):=\frac{u}{\left\langle u, z-z_{0}\right\rangle}$ is a one-to-one mapping from $U$ onto $H$, and it is easy to check that

$$
G(u)=F(y(u)) \text { for every } u \in U .
$$

It follows that $G=c$ on $U$, and since $G(u)+G(-u)=\operatorname{vol}(L)$ for every $u \in S^{n-1}, G=$ $\operatorname{vol}(L)-c$ on $-U$. Now, $S^{n-1} \cap\left(z-z_{0}\right)^{\perp}$ is contained in the closures of both $U$ and of $-U$ in $S^{n-1}$. Since $G$ is continuous on $S^{n-1}, G=c=1-c=\frac{\operatorname{vol}(L)}{2}$ on $S^{n-1}$. The fact that $L$ is centrally symmetric about $z$ now follows by a classical result (see [8] or [6]).

4. Additional comments and some open problems. We know that although $C(L)$ and $I(L, z)$ are not in general convex bodies (by [14], $C(L)$ is convex for $n \leq 3$ and by [2], if $\Delta_{n}$ is the simplex in $\mathbb{R}^{n}, C\left(\Delta_{n}\right)$ is not convex if $\left.n \geq 4\right)$. However $C(L)$ and $I(L, g(L))$, where $g(L)$ is the centroid of $L$, are almost convex, and even almost ellipsoids, in the sense that there exist some constants $c>d>0$, independent on $n$ and $L$, such that for every $u \in S^{n-1}$, one has

$$
\begin{aligned}
\frac{d}{\operatorname{vol}(L)^{\frac{3}{2}}}\left(\int_{L-g(L)}\langle x, u\rangle^{2} d x\right)^{\frac{1}{2}} & \leq \frac{1}{\max _{t} \operatorname{vol}\left(L \cap\left(t u+u^{\perp}\right)\right)}=\rho_{C(L)}(u) \\
& \leq \frac{1}{\operatorname{vol}\left(L \cap u^{\perp}\right)}=\rho_{I(L, g(L))}(u) \\
& \leq \frac{c}{\operatorname{vol}(L)^{\frac{3}{2}}}\left(\int_{L-g(L)}\langle x, u\rangle^{2} d x\right)^{\frac{1}{2}} .
\end{aligned}
$$

In the centrally symmetric case, this was proved by Hensley, and Ball [1] (for sharp constants, see also [19]), and in the general case by Schütt [22] and Fradelizi [7] (the latter with sharp constants). We have seen that $\rho_{I(L, g(L))} \leq \rho_{C I(L, g(L))}$. A natural question to ask now is

Open Problem 1. Does there exist a universal constant $C>0$, independent on the convex body $L$ in $\mathbb{R}^{n}$ and on $n \geq 1$, such that $\rho_{C I(L, g(L))} \leq C \rho_{I(L, g(L))}$ ? Of course, in view of the preceding inequalities, an affirmative answer to this question would say that the radial functions of $C(L), C I(L)$ and $I(L, g(L))$ are all equivalent (with absolute constants).

Observe that an equivalent way of formulating this problem is the following. Let $K$ be a convex body in $\mathbb{R}^{n}$ such that its Santaló point is at 0 . Does there exist an absolute constant $C>0$, independent on $n$ and $K$ such that

$$
\operatorname{vol}\left(\left(P_{u} K\right)^{* P_{u} z}\right) \geq C \operatorname{vol}\left(\left(P_{u} K\right)^{* 0}\right) \text { for every } z \in \operatorname{int}(K) ?
$$


Equivalently, given a convex $M \subset u^{\perp}$, with Santaló point $s(M)$, and a convex body $K$ in $\mathbb{R}^{n}$, with Santaló point $s(K)$, such that $P_{u} K=M$, does

$$
\operatorname{vol}\left(M^{* s(M)}\right) \geq C \operatorname{vol}\left(M^{* P_{u} s(K)}\right)
$$

for some universal constant $C>0$ ?

If one could prove that in this situation, for some universal constant $c>0$, the following is true:

$$
P_{u} s(K)-s(M) \in \frac{c}{n}(M-s(M)),
$$

then an affirmative answer could be given, using the following lemma.

Lemma 8. Let $V$ be a convex body in $\mathbb{R}^{n}$ and $x, y \in \operatorname{int}(V)$. Then

$$
\left(1-\|x-y\|_{V-y}\right)^{n} \operatorname{vol}\left(V^{* x}\right) \leq \operatorname{vol}\left(V^{* y}\right) \leq \frac{\operatorname{vol}\left(V^{* x}\right)}{\left(1-\|y-x\|_{V-x}\right)^{n}}
$$

Proof. One has

$$
\begin{aligned}
\operatorname{vol}\left(V^{* y}\right) & =\operatorname{vol}\left(V^{* y}+y\right)=\operatorname{vol}\left((V-y)^{*}\right) \\
& =\operatorname{vol}\left(\left((V-x-(y-x))^{*}\right)=\int_{(V-x)^{*}} \frac{1}{(1-\langle y-x, z\rangle)^{n+1}} d z\right.
\end{aligned}
$$

because by a formula given in [18], if $L$ is a convex body with 0 in its interior, one has for every $w$ in the interior of $L$,

$$
\operatorname{vol}\left(L^{* w}\right)=\int_{L^{*}} \frac{1}{(1-\langle w, z\rangle)^{n+1}} d z .
$$

Since $\langle y-x, z\rangle \leq\|z\|_{(V-x)^{*}}|| y-x \|_{V-x}$, we get

$$
\operatorname{vol}\left(V^{* y}\right) \leq \int_{(V-x)^{*}} \frac{1}{\left(1-\|z\|_{(V-x)^{*}}|| y-x \|_{V-x}\right)^{n+1}} d z .
$$

Now, if $L$ is a convex body with 0 in its interior, and $0<c<1$, then

$$
\begin{aligned}
\int_{L} \frac{1}{\left(1-c\|z\|_{L}\right)^{n+1}} d z & =n v_{n} \int_{S^{n-1}}\left(\int_{0}^{\frac{1}{\|\theta\|_{L}}} \frac{r^{n-1}}{\left(1-c r\|\theta\|_{L}\right)^{n+1}} d r\right) d \theta \\
& =n v_{n} \int_{S^{n-1}} \frac{1}{n}\left[\left(\frac{r}{1-c r\|\theta\|_{L}}\right)^{n}\right]_{0}^{\frac{1}{\|\theta\|_{L}}} d \theta \\
& =v_{n} \int_{S^{n-1}} \frac{1}{(1-c)^{n}\|\theta\|_{L}^{n}} d \theta=\frac{\operatorname{vol}(L)}{(1-c)^{n}} .
\end{aligned}
$$

From which, together with (3), we get

$$
\operatorname{vol}\left(V^{* y}\right) \leq \frac{\operatorname{vol}\left(V^{* x}\right)}{\left(1-\|y-x\|_{V-x}\right)^{n}}
$$


Applying the same formula while interchanging the roles of $x$ and $y$, one has

$$
\operatorname{vol}\left(V^{* x}\right) \leq \frac{\operatorname{vol}\left(V^{* y}\right)}{\left(1-\|x-y\|_{V-y}\right)^{n}} .
$$

It is well known (see, e.g. [19]) that there is an affine transformation $A: \mathbb{R}^{n} \rightarrow \mathbb{R}^{n}$ such that $A L=M$ is isotropic, that is, it satisfies $\operatorname{vol}(M)=1$ and for every $u \in S^{n-1}$,

$$
\frac{1}{\operatorname{vol}(M)}\left(\int_{M-g(M)}\langle x, u\rangle^{2} d x\right)^{\frac{1}{2}}=L_{M},
$$

where $L_{M}$ is the isotropic constant of $M$. In that context, Problem 1 is equivalent to

Open Problem 2. Let $M$ be an isotropic convex body. Is $C I(M)$ equivalent to the Euclidean ball (with absolute constant independent on $M \subset \mathbb{R}^{n}$ and on $n$ )?

Of course, Problems 1 and 2 are non-trivial only if $L$ or $M$ are not centrally symmetric. The particular case of the simplex is still open.

Open Problem 3. Let $\Delta_{n}$ be a simplex in $\mathbb{R}^{n}$. Is there a constant $c$ independent on $n$ such that for every $u \in S^{n-1}$

$$
\operatorname{vol}\left(\Delta_{n} \cap u^{\perp}\right) \leq c \operatorname{vol}\left(\left(\left(\Delta_{n} \cap u^{\perp}\right)^{* 0}\right)^{* s}\right)=c \operatorname{vol}\left(\left(P_{u}\left(\Delta_{n}^{* g}\right)\right)^{* s}\right) ?
$$

Observe that when $\Delta_{n}$ is a regular simplex inscribed in the Euclidean ball, since $\left(\Delta_{n}\right)^{* 0}=-n \Delta_{n}$, one has

$$
\left(\Delta_{n} \cap u^{\perp}\right)^{* 0}=P_{u}\left(\left(\Delta_{n}\right)^{* 0}\right)=P_{u}\left(-n \Delta_{n}\right)
$$

and thus

$$
\operatorname{vol}\left(\left(\left(\Delta_{n} \cap u^{\perp}\right)^{* 0}\right)^{* s}\right)=\frac{1}{n^{n-1}} \operatorname{vol}\left(\left(P_{u} \Delta_{n}\right)^{* s}\right)
$$

About Problem 3, one may remark that by affine invariance, we may suppose without loss of generality that $\Delta_{n}$ is the regular simplex with vertices $e_{1}, \ldots, e_{n+1},\left|e_{i}\right|=1$, centred at $0=e_{1}+\cdots+e_{n+1}$. For $1 \leq i \neq j \leq n+1$, one has then $\left\langle e_{i}, e_{j}\right\rangle=-\frac{1}{n}$.

FACT. Let $A \subset\{1, \ldots, n+1\}$ be such that $1 \leq k:=\operatorname{card}(A) \leq n$ and define

$$
u_{A}=\frac{\sum_{i \in A} e_{i}}{\left|\sum_{i \in A} e_{i}\right|}=\sqrt{\frac{n}{k(n+1-k)}} \sum_{i \in A} e_{i} \in S^{n-1} .
$$

Then 0 is the centroid of $\Delta_{n} \cap u_{A}^{\perp}$.

It suffices to show that 0 is the Santalo point of $P_{u_{A}} \Delta_{n}$. Let

$$
e_{l}^{\prime}=P_{u_{A}} e_{l}, 1 \leq l \leq n+1, E=\left[e_{i}^{\prime}, i \in A\right] \text {, and } F=\left[e_{j}^{\prime} ; j \notin A\right] .
$$

Then $E$ and $F$ are linear subspaces of $u_{A}^{\perp} \operatorname{such}$ that $\operatorname{dim}(E)=k-1, \operatorname{dim}(F)=n-k$, $\langle x, y\rangle=0$ for every $x \in E$ and $y \in F$, and

$$
S_{A}:=\overline{\operatorname{conv}}\left(\left\{e_{i}^{\prime}, i \in A\right\}\right) \subset E \text { and } T_{A}:=\overline{\operatorname{conv}}\left(\left\{e_{j}^{\prime}, j \notin A\right\}\right) \subset F
$$

are regular simplices with centre of mass at 0 . It follows that 0 is the Santaló point of $S_{A}$ in $E$ and of $T_{A}$ in $F$, when 0 is the Santaló point of $P_{u_{A}} \Delta_{n}=\operatorname{conv}\left(S_{A}, T_{A}\right)$. It 
follows that 0 is the centroid of $\Delta_{n} \cap u_{A}^{\perp}$, which can be described as

$$
\Delta_{n} \cap u_{A}^{\perp}=S_{A}^{*} \times T_{A}^{*},
$$

where $S_{A}^{*}$ and $T_{A}^{*}$ are the polars of $S_{A}$ and of $T_{A}$, respectively, in $E$ and $F$.

A corollary of this result is the following.

Proposition 9. For every $A \subset\{1, \ldots, n+1\}$, such that $1 \leq k:=\operatorname{card}(A) \leq n$, one has

$$
\left\|u_{A}\right\|_{C I\left(\Delta_{n}, 0\right)}=\left\|u_{A}\right\|_{I\left(\Delta_{n}, 0\right)} .
$$

Moreover, in the particular case when $u^{\perp} \cap \Delta_{n}$ is itself a simplex, one has the following computational proposition.

Proposition 10. Let $u \in S^{n-1}$, and if $u=\sum_{i=1}^{n+1} u_{i} e_{i} \in S^{n-1}$ with $\sum_{i=1}^{n+1} u_{i}=0$ and $u_{1}, \ldots, u_{n} \geq 0>u_{n+1}$, then $u^{\perp} \cap \Delta_{n}$ is a simplex and

$$
\rho_{I\left(\Delta_{n}, 0\right)(u)}=\operatorname{vol}\left(\Delta_{n} \cap u^{\perp}\right)=\frac{1}{(n-1) !} \frac{(n+1)^{\frac{n+1}{2}}}{n^{\frac{n}{2}-1}} \frac{1}{\prod_{i=1}^{n}\left(u_{i}+\sum_{j=1}^{n} u_{j}\right)}
$$

and

$$
\left.\rho_{C I\left(\Delta_{n}, 0\right)(u)}=\operatorname{vol}\left(\left(\Delta_{n} \cap u^{\perp}\right)^{* 0}\right)^{* S}\right)=\frac{1}{(n-1) !} \frac{n^{\frac{n}{2}+1}}{(n+1) \frac{n+1}{2}} \frac{1}{\sum_{i=1}^{n} u_{i}} .
$$

It follows from Proposition 10 that $C I\left(\Delta_{n}, 0\right)$ has $2 n+2$ small faces around $u= \pm e_{i}$, $1 \leq i \leq n+1$. Nevertheless, it is easy to check that for such directions $u \in S^{n-1}$ one has

$$
1 \leq \frac{\operatorname{vol}\left(\Delta_{n} \cap u^{\perp}\right)}{\left.\operatorname{vol}\left(\left(\Delta_{n} \cap u^{\perp}\right)^{* 0}\right)^{* s}\right)} \leq \frac{e}{2}
$$

\section{REFERENCES}

1. K. Ball, Logarithmically concave functions and sections of convex sets in $\mathbb{R}^{n}$, Stud. Math. 88 (1988), 69-84. $127-129$.

2. U. Brehm, Convex bodies with non-convex cross-section bodies, Mathematika 46 (1999), $1-12$.

3. H. Busemann, Volume in terms of concurrent cross-sections, Pac. J. Math. 3 (1953),

4. S. Campi and P. Gronchi, Volume inequalities for sets associated with convex bodies, in Integral geometry and convexity (Grinberg E. L., Li S., Zhang G. and Zhou J., Editors) (World Sci. Publ., Hackensack, NJ, 2006), 1-15.

5. S. Campi and P. Gronchi, On volume product inequalities for convex sets, Proc. Am. Math. Soc. 134 (2006), 2393-2402.

6. K. J. Falconer, Applications of a result on spherical integration to the theory of convex sets, Am. Math. Monthly 90 (1983), 690-693.

7. M. Fradelizi, Hyperplane sections of convex bodies in isotropic position, Beiträge Algebra Geom. 40 (1999), 163-183.

8. P. Funk, Über eine geometriche Anvendung der Abelschen Integralgleichung. Math. Ann. 77 (1916), 129-135. 
9. R. J. Gardner, Geometric tomography, in Encyclopedia of mathematics and its applications, Vol. 58, 2nd edn. (Cambridge University Press, Cambridge, UK, 2006).

10. B. Grünbaum, Measures of symmetry for convex sets, in Proceedings of symposia in pure mathematics, Vol. VII (American Mathematical Society, Providence, RI, 1963), 233-270.

11. D. Hensley, Slicing convex bodies-Bounds for slice area in terms of the body's covariance, Proc. Am. Math. Soc. 79 (1980), 619-625.

12. E. Lutwak, Intersection bodies and dual mixed volumes, Adv. Math. 71 (1988), 232-261.

13. E. Makai Jr., H. Martini and T. Ódor, Maximal sections and centrally symmetric bodies, Mathematika 47 (2000), 19-30.

14. M. Meyer, Maximal hyperplane sections of convex bodies, Mathematika 46 (1999), $131-136$

15. M. Meyer and S. Reisner, Characterizations of ellipsoids by section-centroid location, Geom. Dedicata 31 (1989), 345-355.

16. M. Meyer and S. Reisner, Shadow systems and volumes of polar convex bodies, Mathematika 53 (2006), 129-148 (2007).

17. M. Meyer, C. Schütt and E. Werner, A convex body whose centroid and Santaló point are far apart, arXiv:1001.0714v1 (preprint).

18. M. Meyer and E. Werner, The Santaló-regions of a convex body, Trans. A.M.S. 350 (1998), 4569-4591.

19. V. D. Milman and A. Pajor, Isotropic position and inertia ellipsoids and zonoids of the unit ball of a normed $n$-dimensional space, in Geometric aspects of functional analysis (1987-88), Lecture Notes in Math., 1376 (Springer, Berlin, 1989), 64-104.

20. L. A. Santaló, Un invariante afin para los cuerpos convexos del espacio de $n$ dimensiones, Portugal Math. 8 (1949), 155-161.

21. R. Schneider, Convex bodies, the Brunn-Minkowski theory (Cambridge University Press, Cambridge, UK, 1993).

22. C. Schütt, Floating body, illumination body, and polytopal approximation, in Convex geometric analysis (Berkeley, CA, 1996), 203-229; Math. Sci. Res. Inst. Publ. 34 (1999) (Cambridge University Press, Cambridge).

23. G. C. Shephard, Shadow systems of convex sets. Israel J. Math. 2 (1964), 229-236. 